\title{
A modified sliding mode control of a nonlinear methane fermentation process
}

\author{
Plamena Zlateva* \\ Institute of Robotics and Institute of Mathematics and Informatics, Bulgarian Academy of Sciences, Sofia, Bulgaria
}

\begin{abstract}
A continuous methane fermentation process for biogas production is considered. This biogas production process is described by a system of two nonlinear differential equations and one nonlinear algebraic equation. The paper purpose is to propose an approach for designing a modified sliding mode control (so-called binary control) of a nonlinear methane fermentation process. The control design is carried out with direct use of nonlinear model and on-line measurement for two variables only (the concentration of the organic pollutants and biogas production rate). The model of the sliding mode control is developed with respect to an auxiliary input variable in order to obtain the smooth signal of the dilution rate, which is need in the fermentation processes. The state variables, external disturbance, process output and control input are varied in the known intervals. The asymptotic output stabilization problem is solved. The good system robustness with the designed modified sliding mode control (the binary control) about various disturbances is proved through simulation investigations in MATLAB using Simulink.
\end{abstract}

\section{Introduction}

Nowadays, a very useful source of green energy is biogas $[1,2]$. Worldwide, there is increasing interest in its wider use as an environmental fuel [3-6].

The biogas production is done by anaerobic digestion (oxygen-free environment) of various organic material with the help of the methanogenic bacteria [4-7]. This methane fermentation process generally carried out in continuously stirred tank bioreactors [8].

The methane fermentation process is usually described by a system from ordinary differential equations with uncertain parameters. For these reason it is needed the developing of sophisticated control algorithms based on nonlinear models [9-11].

It is known that the sliding mode control is effectively used in the stabilization of nonlinear and uncertain processes $[12,13]$. The guaranteed system invariance to parameter uncertainties and external disturbances is the main advantage of the sliding mode control [14-16]. However, the control signal is discontinuous in time, which leads to chattering phenomenon. In practice, such control is hard to realize therefore various techniques for chattering attenuation are proposed (boundary layers, auxiliary input variable, binary control, input-dependent sliding surface) $[17,18]$.

The paper purpose is to propose an approach for designing a modified sliding mode control (so-called binary control) of a nonlinear methane fermentation process. The control design is carried out with direct use of nonlinear model. The model of this sliding mode control is developed with respect to an auxiliary variable in order to obtain the smooth signal of the dilution rate.

\section{A mathematical model of the methane fermentation process}

In this study, a simplified one-step reaction scheme is used to describe the continuous process of methane fermentation (the biogas production process):

$$
S+X \longrightarrow X+Q
$$

where $S$ is the substrate concentration (the concentration of organic pollutants), $[\mathrm{g} / \mathrm{l}] ; X$ - the biomass concentration (the methanogenic bacteria), $[\mathrm{g} / \mathrm{l}]$; $Q$ - the biogas production rate, [g/(l.day)].

The continuous fermentation dynamics in a stirred tank bioreactor (methane tank) corresponding to reaction scheme (1) are described by a system of two nonlinear differential equations and one nonlinear algebraic equation. This mathematical model is written as follows:

$$
\begin{aligned}
& \frac{d X(t)}{d t}=\mu(t) X(t)-D(t) X(t) \\
& \frac{d S(t)}{d t}=-k_{1} \mu(t) X(t)+D(t)\left(S_{\text {in }}-S(t)\right) \\
& Q(t)=k_{2} \mu(t) X(t)
\end{aligned}
$$

where $S_{\text {in }}[\mathrm{g} /($ l.day) $]$ is the influent substrate concentration (the concentration of the polluting organics); $D(t)$, [1/day] is the dilution rate for the polluting organics with concentration $S_{\text {in }}$ added into the bioreactor; $\mu(t),[1 /$ day $]$ - the specific growth rate of the biomass (the methanogenic bacteria); $k_{l}$ - the yield coefficient; $k_{2}$ - the coefficient of proportionality.

\footnotetext{
*Corresponding author: plamzlateva@ abv.bg
} 
Here, for biological and technological reasons, the following assumptions are made about the methane fermentation process:

a1). The state variables - the biomass $X(t)$ and substrate $S(t)$ concentrations are positive, bounded and differentiable functions of time:

$$
0<X^{\min } \leq X(t) \leq X^{\max } \text { and } 0<S^{\min } \leq S(t) \leq S^{\min },
$$

where $X^{\min }, X^{\max }, S^{\min }$ and $S^{\max }$ are known constants;

a2). The biogas production rate $Q(t)$ is a positive, bounded and differentiable function of time

$$
0 \leq Q^{\min } \leq Q(t) \leq Q^{\max },
$$

where $Q^{\min }$ and $Q^{\max }$ are known constants;

a3). The process input is the dilution rate, $u(t)=D(t)$. It is a differentiable function of time and varied in known boundaries:

$$
0 \leq D^{\min } \leq D(t) \leq D^{\max },
$$

where $D^{\min }$ and $D^{\max }$ are known constants;

a4). The specific growth rate is known, nonlinear and differentiable function of time, depending on the state variables and the kinetics coefficients:

$$
\mu(t)=\mu(X(t), S(t), p)
$$

where $p$ is a vector of the kinetic coefficients;

a4). The influent substrate concentration $S_{\text {in }}$ is an external disturbance. It is varied in known boundaries:

$$
0<S^{\max }<S_{\text {in }}^{\min } \leq S_{\text {in }} \leq S_{\text {in }}^{\max },
$$

where $S_{i n}^{\min }$ and $S_{i n}^{\max }$ are known constants; $S^{\max }$ is the maximum admissible value of the substrate concentration $S(t)$

a5). Only the concentration of the organic pollutants $S(t)$ and the biogas production rate $Q(t)$ are measured online. The data for both process variables are obtained by direct measurements or by software sensors.

a6). The process output is the biogas production rate $y(t)=Q(t)$.

\section{Design of a modified sliding mode control for nonlinear methane fermentation process}

\subsection{Problem statement for control design}

In this study, the problem is to propose an approach for designing of a modified sliding mode control (the socalled binary control) of the continuous process for the production of biogas (methane fermentation) by energy criterion, i.e. the target product is the biogas.

In particular, the problem is to stabilize the output, $y(t)$ $=Q(t)$ at different desired set-points $g=Q^{*}$ by using the dilution rate $u(t)=D(t)$ as control input, while transients processes satisfy a certain quality.

The design of the modified sliding mode control is carried out with using on-line measured data only for the two variables - the output variable $y(t)=Q(t)$ and the state variable $S(t)$. The control model is derived with direct used of nonlinear model (2)-(4). The deigned control guaranties the robustness of the nonlinear system on various internal and external disturbances. It is also guaranteed the invariance of the output variable $y(t)=Q(t)$ in regard to immeasurable external disturbance $S_{i n}(t)$.

The form of the static process characteristic $y(u)=Q(D)$ is retained in the various non-linear mathematical models for the specific growth rate of biomass. The static process characteristic $y(u)=Q(D)$ is a nonlinear and limited function that has a maximum $Q^{\max }\left(D_{\max }\right)$. This peculiarity also predetermines the specific requirements of the control model. It is necessary to point out that the regulation of the system output about the set-point variations is especially difficult, when the process initial state is to the right of the maximum of the function $D(0)>D_{\max }$.

\subsection{Transformation of the nonlinear model}

Here, a suitable transformation of the nonlinear system of two first order differential equations is performed in order to stabilize the output variable $y(t)=Q(t)$ around the given set-point $g=Q^{*}\left(D^{*}\right)$.

As a first step, the nonlinear model (2)-(4) is reduced to a second-order differential equation by differentiating the output twice:

$$
\begin{aligned}
\ddot{Q}(t)=\left(\frac{\ddot{\mu}(t)}{\mu(t)}+\right. & \left.2 \dot{\mu}(t)-\frac{\dot{\mu}(t)}{\mu(t)} D(t)\right) Q(t)+ \\
& +(\mu(t)-D(t)) \dot{Q}(t)+-\dot{D}(t) Q(t) .
\end{aligned}
$$

Suppose it is desired to stabilize the process output $y(t)=Q(t)$ to its set-point $g=Q^{*}\left(D^{*}\right)$. For this reason, the output error $e(t)$ and the input control action $u(t)$ are defined as follows:

$$
e(t)=Q(t)-Q^{*}=y(t)-g \text { and } u(t)=D(t)-D^{*},
$$

where $g=Q^{*}$ and $D^{*}$ are positive constants.

The following auxiliary input variable is defined:

$$
v(t)=\dot{u}(t)=\left(D(t)+D^{*}\right)^{\prime}=\dot{D}(t)
$$

in order to avoid the chattering phenomenon and to obtain a smoothed input signal. The model of the sliding mode is designed with respect to this auxiliary input variable. In particular, the sliding mode is realized with respect to the derivative of the control input $D(t)$.

The following new state variables and a vector are introduced: $x_{1}(t)=e(t) ; x_{2}(t)=\dot{e}(t)$ and $x(t)=\left[x_{1}(t), x_{2}(t)\right]^{T}$.

For the design purposes of the modified sliding mode control (the binary control), the model of the methane fermentation process (2)-(4), taking into account (10)-(12), is represented by the following generalized observational canonical form [12]: 


$$
\begin{aligned}
& \frac{d x_{1}(t)}{d t}=x_{2}(t) \\
& \frac{d x_{2}(t)}{d t}=F(x(t), u(t), v(t))
\end{aligned}
$$

where

$$
\begin{gathered}
F(t)=\left(\frac{\ddot{\mu}(t)}{\mu(t)}+2 \dot{\mu}(t)-\frac{\dot{\mu}(t)}{\mu(t)}\left(u(t)+D^{*}\right)\right)\left(x_{1}(t)+Q^{*}\right)+ \\
+\left(\mu(t)-\left(u(t)+D^{*}\right)\right) x_{2}(t)-\left(x_{1}(t)+Q^{*}\right) v(t)
\end{gathered}
$$

The function $F(x(t), u(t), v(t))$ is rewritten as follows:

$$
F(x(t), u(t), v(t))=f(x(t), u(t))+b(x(t)) \cdot v(t),
$$

where

$$
\begin{aligned}
& f(x(t), u(t))=\left(\frac{\ddot{\mu}(t)}{\mu(t)}+2 \dot{\mu}(t)-\frac{\dot{\mu}(t)}{\mu(t)}\left(u(t)+D^{*}\right)\right)\left(x_{1}(t)+Q^{*}\right)+ \\
& +\left(\mu(t)-\left(u(t)+D^{*}\right)\right) x_{2}(t) \\
& b(x(t))=-\left(x_{1}(t)+Q^{*}\right) .
\end{aligned}
$$

\subsection{Model of the modified sliding mode control}

Here, the closed control system (13)-(15) is assumed to have the desired properties if, at any given moment in time, the new state variables are connected by the linear dependence $\sigma(t)$, which defines the sliding surface:

$$
\sigma(t)=\sigma(x(t))=c x(t)=c_{1} x_{1}(t)+c_{2} x_{2}(t)=0,
$$

where $c=\left[c_{1}, c_{2}\right] ; c_{1}=$ const $>0, c_{2}=$ const $>0$.

The problem of stabilization of the closed loop system is reformulated to guarantee equality $\sigma(t)=0$.

It is well known that the control input signal, dilution rate $D(t)$, in fermentation processes has to be smooth. This come from the fact that the fermentation processes involve living microorganisms. For this reason, the model of the proposed sliding mode control (the binary control) is designed with respect to the auxiliary input variable $v(t)$ as follow:

$$
\begin{aligned}
& v(t)=\gamma(t) \cdot V_{M}(t) \\
& \frac{d \gamma(t)}{d t}=-\varphi(t) \operatorname{sgn}(\sigma(t)),
\end{aligned}
$$

where $\gamma(t)$ is a real variable and $|\gamma(t)| \leq 1 ; V_{M}(t)$ and $\varphi(t)$ are nonlinear positive functions.

According to the theory of the sliding mode control, and in particular the binary control theory [18], in order to guarantee the asymptotic stability of a closed system (13)-(15), the functions and must satisfy the following conditions:

$$
V_{M}(t) \operatorname{sgn}(\sigma(t) \gamma(t))<-\sup _{t}\left|\frac{\tilde{c}_{1} x_{2}(t)+\tilde{c}_{2} f(t)}{\tilde{c}_{2} b(t)}\right|
$$

where $\tilde{c}_{1}=c_{1} \pm \delta \beta_{1}>0, \tilde{c}_{2}=c_{2} \pm \delta \beta_{2}>0$ when $\tilde{c}_{2} b(t)>0$;

$$
0<\delta<1 ; \quad 0<\beta_{1} \leq 1 ; \quad 0<\beta_{2} \leq 1 ;
$$

and

$$
\varphi(t) \geq \frac{2}{\delta} \sup _{t}\left|\frac{\tilde{c}_{1} x_{2}(t)+\tilde{c}_{2}\left(f(t)+b(t) \gamma(t) V_{M}(t)\right)}{\beta_{1} x_{1}(t)+\beta_{2} x_{2}(t)}\right|
$$

where $\tilde{c}_{1}=c_{1} \pm \delta \lambda \beta_{1}>0, \quad \tilde{c}_{2}=c_{2} \pm \delta \lambda \beta_{2}>0,0 \leq \lambda \leq 1$.

\section{Simulation investigations}

Several simulation investigations in MATLAB using Simulink are carried out to demonstrate the effectiveness of the designed modified sliding mode control to stabilize a nonlinear methane fermentation process for biogas production in presence of uncertainty and disturbances.

There are many nonlinear functions to describe the specific growth rate of the biomass (the methanogenic bacteria) limited by a single substrate. However, Monodtype kinetics are widely used to express the function of the specific reaction rate:

$$
\mu(t)=\frac{\mu_{m} S(t)}{k_{s}+S(t)},
$$

where $\mu_{m}$ is the maximum specific growth rate and $k_{s}$ the saturation coefficient. In this case, the kinetic coefficients vector is written as $p=\left(\mu_{m}, k_{s}\right)$.

Here, the static characteristic function $y(u)=Q(D)$ is defined as follows

$$
Q(D)=\frac{k_{2}}{k_{1}}\left(S_{i n}-\frac{k_{s} \cdot D}{\mu_{m}-D}\right) D .
$$

The following parameter values of the methane fermentation process are adopted:

$$
\mu_{m}=0.4 ; \quad k_{s}=0.4 ; \quad k_{1}=27.3 ; \quad k_{2}=75 .
$$

The external disturbance (the influent substrate concentration) has a nominal value $S_{i n}=3$. However, it is varied in known boundaries: $2 \leq S_{\text {in }} \leq 4$.

The maximum of the static characteristic function (21) is calculated for the given values of the process parameter and external disturbance. The following values for the biogas production rate and dilution rate are obtained: $Q^{\max }=1.613$ and $D^{\max }=0.263$.

In this case, the functions $f(t)$ and $b(t)$ are as follows:

$$
\begin{gathered}
f(t)=\left(\frac{\mu_{m} S(t)}{k_{s}+S(t)}-\left(u(t)+D^{*}\right)-\frac{k_{1} k_{s} Q(t)}{k_{2}\left(k_{s}+S(t)\right) S(t)}\right) x_{2}(t)+ \\
+2\left(-\frac{\left(u(t)+D^{*}\right) \dot{S}(t)}{\left(k_{s}+S(t)\right) S(t)}-\frac{k_{s}(\dot{S}(t))^{2}}{\left(k_{s}+S(t)\right)^{2} S(t)}+\frac{\mu_{m} k_{s} \dot{S}(t)}{\left(k_{s}+S(t)\right)^{2}}\right)\left(x_{1}(t)+g\right) \\
b(t)=\left(\frac{k_{s}\left(S_{\text {in }}-S(t)\right)}{\left(k_{s}+S(t)\right) S(t)}-1\right)\left(x_{1}(t)+g\right) .
\end{gathered}
$$


For simulation investigations, the following values for the parameters of the designed control (the modified sliding mode control) are selected:

$$
\beta_{1}=1 ; \quad \beta_{2}=1 ; c_{1}=0.5 ; \quad c_{2}=1 ; \quad \delta=0.01 ; \quad \lambda=0.5 .
$$

The simulation results obtained from the step changes of the set-point to the left of the maximum value, $Q^{\max }$.

$$
Q^{*}=g=1.137 \rightarrow 1.429 \rightarrow 1.603 \rightarrow 1.484,
$$

are presented in Fig. 1. In this case, the following initial values of the variables are selected:

$$
D(0)=0.15 ; \quad S(0)=0.24 ; \quad X(0)=0.101
$$

The good output behavior of the nonlinear methane fermentation process and the smooth control input $u(t)=D(t)$ are evident when the set-point $\left(g=Q^{*}\right)$ is changed to the left of the maximum value, $Q^{\max }=1.613$. In this case, the values of control input are also to the left to the $D_{\max }, D(t)>D_{\max }$.
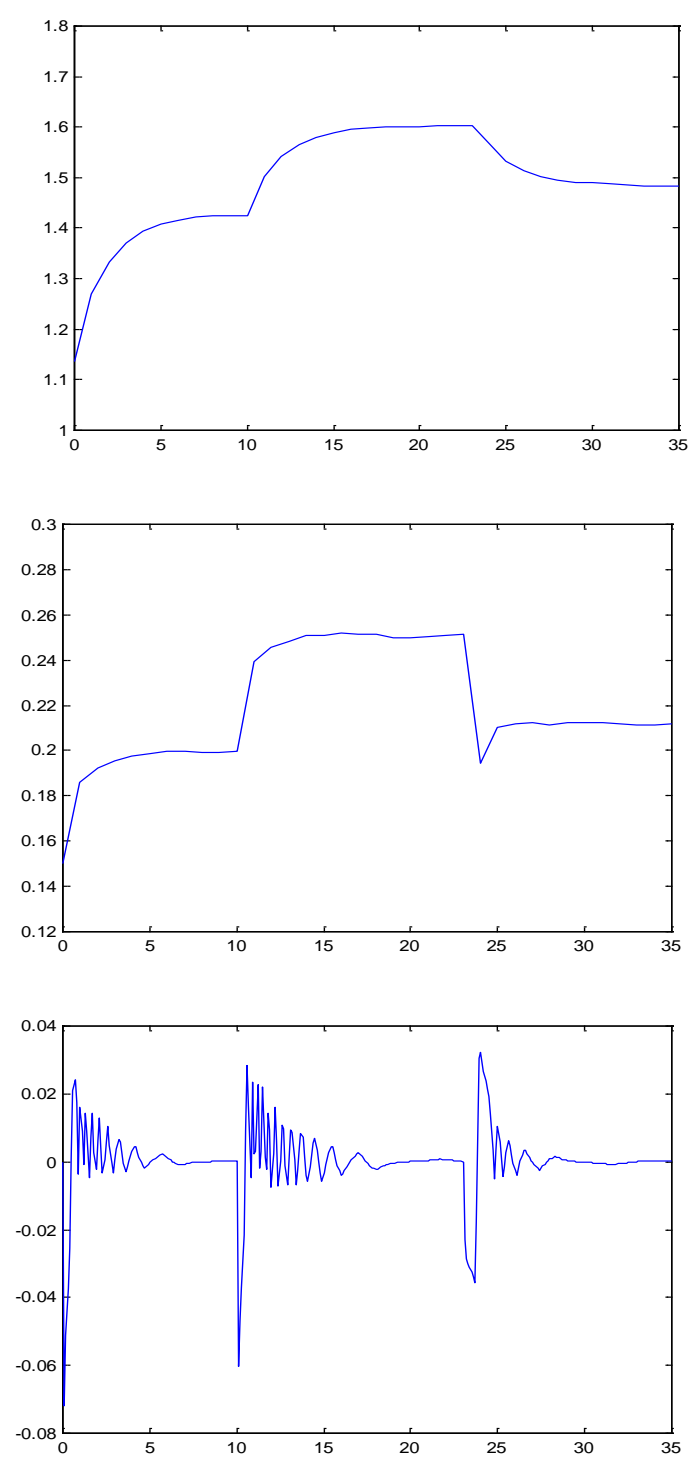

Fig. 1. Process behavior when the set-point, $g=Q^{*}$ is changed to the left of the maximum value $Q^{\max }$ : the curve of $Q(t), D(t)$ and $v(t)$, respectively.
Figure 2 presents the simulation results obtained from the step changes of the set-point around the maximum value of the output, $Q^{\text {max }}$.

$$
Q^{*}=g=1.484 \rightarrow 1.613 \rightarrow 1.484,
$$

with corresponding values of the control input (the dilution rates):

$$
D^{*}=0.3 \rightarrow 0.263 \rightarrow 0.3 .
$$

In this case, the initial state of the output is to the right of the maximum value.

It can be seen that the modified sliding mode control successfully responds to the set-point changes, providing the desired aperiodic transition and good performance.
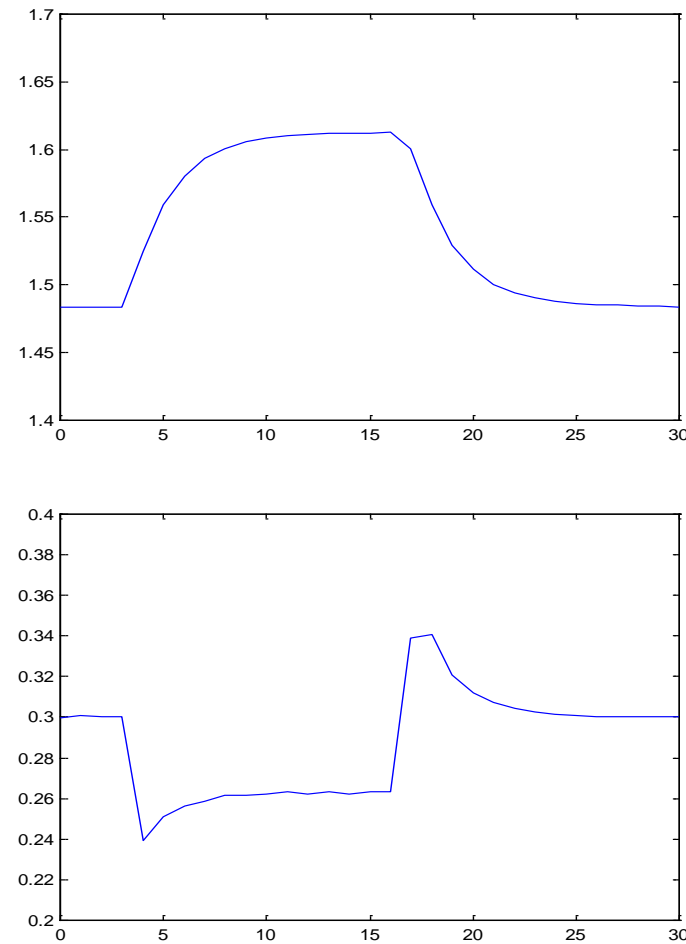

Fig. 2. Process behavior when the set-point, $g=Q^{*}$ is changed around of the maximum value $Q^{\max }$ : the curve of $Q(t)$ and $D(t)$, respectively.

Figure 3 presents the simulation results obtained from the step changes of the external disturbance (influent concentration of the polluting organics) $S_{\text {in }}$ :

$$
S_{\text {in }}=3.5 \rightarrow 2.5 \rightarrow 3.5 \rightarrow 3,
$$

where $Q^{*}(t)=1$ is set-point of the biogas production rate.

The simulation results clearly demonstrate the invariance of the process output variable $y(t)=Q(t)$ with respect to external disturbance $S_{\text {in }}$. 

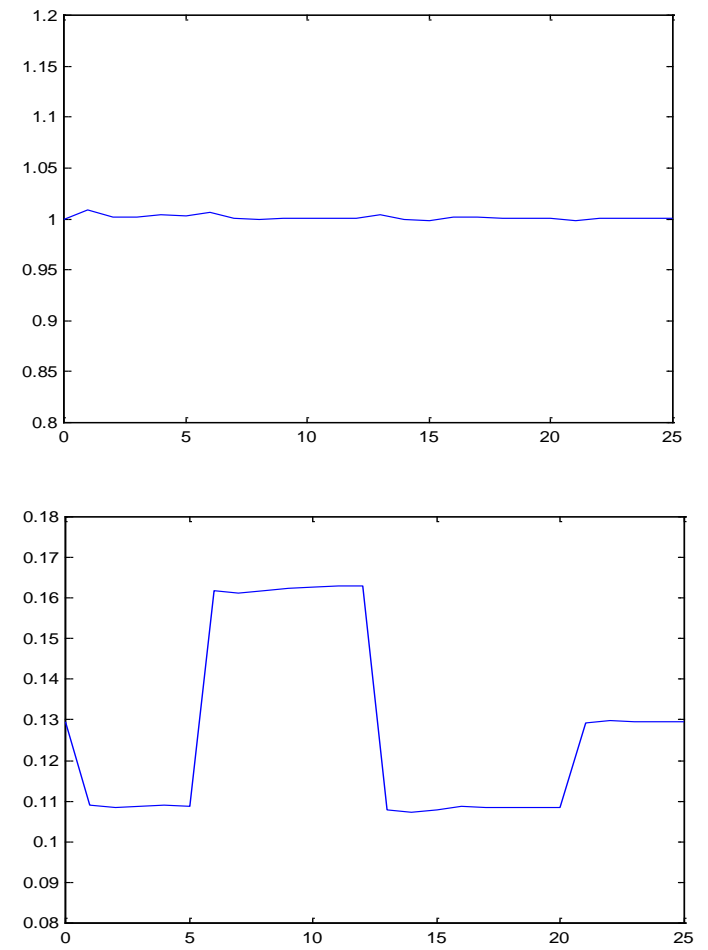

Fig. 3. Process behavior when the set-point, $g=Q^{*}$ is constant and the external disturbance $S_{i n}$ is changed: the curve of $Q(t)$ and $D(t)$, respectively.

\section{Conclusions}

The modified sliding mode control (so-called binary control) of a nonlinear methane fermentation process for biogas production is proposed. The control design is carried out with direct use of nonlinear model and on-line measurement for two variables only (the concentration of the organic pollutants and biogas production rate). The model of the sliding mode control is developed with respect to an auxiliary input variable in order to obtain the smooth signal of the dilution rate, which is need in the fermentation processes. The state variables, external disturbance, process output and control input are varied in the known intervals. The good system robustness with the designed modified sliding mode control (the binary control) about various disturbances is proved through simulation investigations in MATLAB using Simulink.

This paper is partially supported by the National Scientific Program "Information and Communication Technologies for a Single Digital Market in Science, Education and Security (ICTinSES)", contract No D01-205/23.11.2018, financed by the Ministry of Education and Science in Bulgaria.

\section{References}

1. F. van Foreest, Perspectives for Biogas in Europe, (Oxford Institute for Energy Studies, 2012)

2. A Wellinger, JD Murphy, D Baxter, The biogas handbook: science, production and applications (IEA Bioenergy, 2013)

3. N. Scarlat, J.-F. Dallemand, Fernando Fahl, Renewable Energy, 129, Part A, 457-472 (2018)

4. REN21, Renewable Energy Policy Network for the 21st Century (2016)

5. S. Achinas, V. Achinas, G. J. W. Euverink, Engineering, 3 (3), 299-307 (2017)

6. Clarke-energy - A Kohler Company, Biogas \& Engines, (www.clarke-energy.com, 2011)

7. Deutsche Energie-Agentur GmbH (DENA), German Energy Agency Biogaspartner Project (https://www.biogaspartner.de/startseite, 2017)

8. B.D. Wellinger, J. Murphy, The Biogas Handbook. Science, Production and Applications (Woodhead Publishing 2013)

9. N. Dimitrova, M. Krastanov, Int. J. Robust. Nonlinear, (wileyonlinelibrary.com, 2011)

10. Mazenc, F., J. Harmand, H. Mounier, NOLCOS (Toulouse, France, 2013)

11. I. Simeonov, E. Chorukova, S. Diop, N. Christov, Termotehnica 2, 8 (2014)

12. P. Zlateva, Bioprocess Engineering 16 (1), 383-387 (1997)

13. Y. Zheng, et al., Mathematical Problems in Engineering 2015, Article ID 319495 (2015)

14. Utkin V. Sliding modes in optimization and control problems (Springer-Verlag, New York, 1992)

15. Y. Shtessel, C. Edwards, L. Fridman, A. Levant, Sliding mode control and observation (Springer, Basel, 2014)

16. L. Fridman, J.-P. Barbot, F. Plestan, Recent Trends in Sliding Mode Control (CPI Group Ltd, 2016)

17. Shang Shi, et al., Int. J. Robust and Nonlinear Control 29 (1), 224-237 (2018)

18. P. Zlateva, Bioprocess Engineering 23 (1), 81-87 (2000) 